
\title{
$\begin{array}{ll}\text { Research Square } & \begin{array}{l}\text { Preprints are preliminary reports that have not undergone peer review. } \\ \text { They should not be considered conclusive, used to inform clinical practice, } \\ \text { or referenced by the media as validated information. }\end{array}\end{array}$
}

\section{An In-Silico Analysis of Acquired Antimicrobial Resistance Genes in Pseudomonas Aeruginosa Plasmids}

\author{
Rashid Ramazanzadeh ( $\square$ atrop_t51@yahoo.com ) \\ Ardabil University of Medical Sciences
}

\section{Software article}

Keywords: Pseudomonas aeruginosa, plasmids, AMR gene

Posted Date: September 15th, 2021

DOl: https://doi.org/10.21203/rs.3.rs-870436/v1

License: (1) This work is licensed under a Creative Commons Attribution 4.0 International License. Read Full License 


\section{Abstract}

Introduction: The aim of this study to reveal the prevalence of acquired antimicrobial genes in sequences of $P$. aeruginosa plasmids by using in silico methods.

Methods: This study included 828 items with using 'Pseudomonas aeruginosa and plasmid' keywords for searching in NCBI database. The sequences of 94 plasmids were retrieved from GenBank and analysed for detection of acquired antimicrobial resistance (AMR) genes with using ResFinder 2.1 database/webserver, KmerResistance 2.2 database. and ResFinderFG 1.0. Plasmids Sequence were aligned with using MEGA X Molecular Evolutionary Genetics Analysis across Computing Platforms.

Results: 67 out of 94 plasmids sequences were qualified as AMR containing plasmids. For detected 9 classes of AMR genes, aminoglycosides $39.6 \%$ were highest rate. The next frequencies were beta-lactams (19\%), sulphonamides (14.5\%), and fluoroquinolones (10.1\%). For nine classes of antibiotics, 74 AMR gene were identified. Prevalent of sulphonamide resistance gene Sul1 was 32 out of 277 gene. In AMR plasmids, 6 ARD family were detected. 15 representative genomic sequences selected from each clade and three clades were revealed from them. The relationship of clades with drug resistance was not significant ( $p$-value $=0.682$ ).

Conclusions: Analyzing the Information in annotated sequence is reveal the mechanism of spreading of resistance gene in plasmids. Detection the trace of AMR genes in world papulation can help to find more response to question in regards to spread of infection and analysis of AMR gene sequences give more insight to scientist to control of Pseudomonas aeruginosa infections.

\section{Introduction}

Pseudomonas aeruginosa habitats are very wide including soil and aquatic environments. This species can cause diseases in humans, animals and plants. $P$. aeruginosa is highly prone to drug resistance mostly by means of plasmids. plasmids are tools for horizontal gene transfer (HGT), in bacterial genetics evolution and adaptation. These mobile genetic elements are very diverse and have a high range hosts and ecological niches. Based on genetic organization, size and host range, 14 incompatibility groups of plasmids have been detected in Pseudomonas[1, 2]. Incompatible groups are using same replication control. This groups are very mobile and capable to gene transfer called promiscuous. Promiscuity gave great role in antibiotic resistance spreading among bacterial populations [3].

Antimicrobial resistance (AMR) is a more growing issue in $P$. aeruginosa infections that cause health system crisis. The empirical use of antibiotics in veterinary and medicine promote and creating resistance plasmids that circulate in universe populations. Spread of plasmid harbor AMR genes as threat to public health and environment leads to mortality and morbidity. Antimicrobial resistance (AMR) in $P$. aeruginosa is a worldwide health problem and need more attention in diagnosis and finding the map of spreading by plasmids. We aimed in this study to reveal the prevalence of acquired antimicrobial genes in sequences of $P$. aeruginosa plasmids by using in silico methods.

\section{Materials And Methods}




\section{Plasmid selection}

This study included all 'Pseudomonas aeruginosa plasmid. Overlay 828 item were found with using 'Pseudomonas aeruginosa and plasmid' keywords for searching in NCBI database (Table 1). Based on web data this sequence has been published from 1991 until 2020. Size of plasmid ranged between $2140 \mathrm{bp}$ 555265 bp and GC content were $30.1-65.8 \%$.

\section{Screening for acquired AMR genes in plasmids}

The sequences of 94 plasmids were retrieved from GenBank. This sequence were conducted in silico analysis for detection of acquired antimicrobial resistance genes with using ResFinder 2.1 database/webserver which 67 plasmids has AMR genes (Table 1) [4]. Fifteen classes of antibiotic presented in database considered for screening that included, aminoglycoside (AG), beta-lactam, colistin, a fluoroquinolone (FQ), fosfomycin, fusidic acid, glycopeptide, macrolide-lincosamide-streptogramin B (MLS), nitroimidazole, oxazolidinone, phenicol, rifampicin (RP), sulphonamide (SM), tetracycline (TC), and trimethoprim (TP). Search setting parameters for all 15 -drug classes were adjusted to $90 \%$ as a minimum for percent identity and $100 \%$ for perfect alignment, also $60 \%$ for minimum length of sequence nucleotides to overlap with resistant genes. DNA of plasmid were submitted into database online software.[5] Analysis results including, predicted phenotype of resistance gene, database accession number, starting contig position of the gene, and alignment highscoring segment pair (HSP) query length, were gathered from program. All date were recorded in excel software. For all plasmids the index of potential multiple antibiotic resistance (p-MAR) were calculated based on screened 15 classes of antibiotics [6,7]. The analysis of p-MAR data's, can classify plasmids into three groups as: multidrug-resistant (MDR), extensively drug-resistant (XDR) or pan drug-resistant (PDR) by using the previously reported standards $[5,8]$ This index is a good marker for epidemiology detection of isolates origin antibiotic use and the rate of 0.2 indicates a 'high-risk' of social health contamination $[6,9]$.

\section{Verification of acquired AMR genes in Aeromonas plasmids}

Further assessments of plasmid containing AMR genes were done with KmerResistance 2.2 database [10, 11]. Analysis was conducted with default setting including, $70 \%$ for identity threshold and $10 \%$ for depth correction. Results were saved on excel sheet and compared with the results of ResFinder program.

\section{Probing of plasmids for antibiotic resistance determinants (ARD)}

The resistance phenotype of gene were explored for plasmids with AMR genes with ResFinderFG 1.0 [12] server, that screened resistance with functional metagenomic antibiotic resistance determinants. This database server setting was considered $98 \%$ for per cent identity and $60 \%$ for minimum query length. Results which screened for more than 13, ARD families were saved in excel sheet as 'assembled contigs/genomes and sequences.

\section{Phylogenetic analysis of the retrieved Plasmids genomes}

Plasmids Sequence were aligned with using MEGA X Molecular Evolutionary Genetics Analysis across Computing Platforms (MEGA X; https://www.megasoftware.net/). software by ClustalW approach. 
phylogenetic tree was done by a maximum parsimony (MP) approach in MEGA X software. Support value were considered 1,000 bootstrap replicates.

\section{Statistical Analysis}

Correlation between GC\% and plasmid size were qualified with using XLSTAT with principal component analysis (PCA) analysis with setting included mean, standard deviation, and correlation (Pearson).

Connection of plasmid with containing AMR gene with sequences available in NCBI databases and similarity between them were determined using BLAST online tool. Sequences were aligned with MEGA X using likelihood model in considering bootstrap procedure (1000 replicates).

\section{Results}

\section{Size and GC content of Pseudomonas plasmids}

PCA were used to determine the relation between size and GC content, of 67 plasmids carrying AMR genes. Size of plasmid ranged between 2140 bp _ 555265 bp and GC content were 30.1-65.8\%. 
Table 1

Pseudomonas aeruginosa Plasmids contain AMR genes

\begin{tabular}{|c|c|c|c|c|}
\hline $\begin{array}{l}\text { strain } \\
\text { number }\end{array}$ & Organism- plasmid & $\begin{array}{l}G+C \\
\%\end{array}$ & size bp & AMR \\
\hline PA1 & Pseudomonas aeruginosa plasmid pP6qnrS1 & 51 & 117945 & yes \\
\hline PA2 & Pseudomonas aeruginosa strain C plasmid pKLC102 & 60.9 & 103532 & yes \\
\hline PA3 & Pseudomonas aeruginosa plasmid pKLC102 & 60.9 & 103532 & yes \\
\hline PA4 & Pseudomonas aeruginosa strain NK546 plasmid pNK546b & 57.1 & 232884 & yes \\
\hline PA5 & Pseudomonas aeruginosa plasmid pUM505 & 60.9 & 123322 & yes \\
\hline PA6 & Pseudomonas aeruginosa strain IP40a plasmid pIP40 & 51.3 & 167554 & yes \\
\hline PA7 & Pseudomonas aeruginosa plasmid pR31014-IMP & 56.4 & 374000 & yes \\
\hline PA8 & Pseudomonas aeruginosa plasmid p12939-PER & 57.3 & 496436 & yes \\
\hline PA9 & Pseudomonas aeruginosa plasmid pJB12 & 62.6 & 30361 & yes \\
\hline PA10 & Pseudomonas aeruginosa strain ST308 plasmid pCOL-1 & 60.2 & 31529 & yes \\
\hline PA11 & Pseudomonas aeruginosa plasmid pA681-IMP & 56.4 & 397519 & yes \\
\hline PA12 & Pseudomonas aeruginosa plasmid p727-IMP & 56.4 & 430173 & yes \\
\hline PA13 & Pseudomonas aeruginosa plasmid pJB35 & 62.8 & 31166 & yes \\
\hline PA14 & Pseudomonas aeruginosa plasmid Rms149 & 59.5 & 57121 & yes \\
\hline PA15 & $\begin{array}{l}\text { Pseudomonas aeruginosa strain FFUP_PS_37 plasmid } \\
\text { pJB37 }\end{array}$ & 57.2 & 464804 & yes \\
\hline PA16 & Pseudomonas aeruginosa strain P378 plasmid P378-IMP & 50.5 & 51207 & yes \\
\hline PA17 & Pseudomonas aeruginosa strain 10265 plasmid p10265-KPC & 58.2 & 38939 & yes \\
\hline PA18 & Pseudomonas aeruginosa strain PA1280 plasmid pICP-4GES & 64.1 & 50914 & yes \\
\hline PA19 & Pseudomonas aeruginosa strain 60512 plasmid p60512-IMP & 62.7 & 24306 & yes \\
\hline PA20 & Pseudomonas aeruginosa strain HS87 plasmid pHS87a & 62.9 & 26825 & yes \\
\hline PA21 & Pseudomonas aeruginosa strain COL-1 plasmid pNOR-2000 & 62.8 & 21880 & yes \\
\hline PA22 & Pseudomonas aeruginosa strain AR441 plasmid unnamed2 & 60.9 & 57052 & yes \\
\hline PA23 & $\begin{array}{l}\text { Pseudomonas aeruginosa strain AR_0356 plasmid } \\
\text { unnamed1 }\end{array}$ & 60.9 & 57053 & yes \\
\hline PA24 & Pseudomonas aeruginosa strain PB353 plasmid pPB353_1 & 57.3 & 59923 & yes \\
\hline PA25 & $\begin{array}{l}\text { Pseudomonas aeruginosa strain PAcoop101 plasmid } \\
\text { pCOOP-101 }\end{array}$ & 62.3 & 26108 & yes \\
\hline PA26 & Pseudomonas aeruginosa strain S04 90 plasmid & 57.7 & 159187 & yes \\
\hline
\end{tabular}




\begin{tabular}{|c|c|c|c|c|}
\hline $\begin{array}{l}\text { strain } \\
\text { number }\end{array}$ & Organism- plasmid & $\begin{array}{l}G+C \\
\%\end{array}$ & size bp & AMR \\
\hline PA27 & $\begin{array}{l}\text { Pseudomonas aeruginosa strain } 15.2986 \text { plasmid } \\
\text { pPSTRAS1 }\end{array}$ & 56.5 & 9910 & yes \\
\hline PA28 & $\begin{array}{l}\text { Pseudomonas aeruginosa strain PA41437 plasmid pOXA- } \\
198\end{array}$ & 60.5 & 48978 & yes \\
\hline PA29 & $\begin{array}{l}\text { Pseudomonas aeruginosa strain PAB546 plasmid pNK546- } \\
\text { KPC }\end{array}$ & 57.2 & 475027 & yes \\
\hline PA30 & Pseudomonas aeruginosa strain PA34 plasmid pMKPA34-1 & 57.2 & 95404 & yes \\
\hline PA31 & Pseudomonas aeruginosa strain 14057 plasmid p14057-KPC & 59.2 & 51663 & yes \\
\hline PA32 & Pseudomonas aeruginosa strain HN39 plasmid pHN39-SIM & 56.9 & 282042 & yes \\
\hline PA33 & Pseudomonas aeruginosa strain HS87 plasmid pHS87b & 60.7 & 11242 & yes \\
\hline PA34 & $\begin{array}{l}\text { Pseudomonas aeruginosa strain PABL048 plasmid } \\
\text { pPABL048 }\end{array}$ & 56.6 & 141954 & yes \\
\hline PA35 & Pseudomonas aeruginosa strain $\mathrm{BH}$ 9 plasmid pBH6 & 63.5 & 41024 & yes \\
\hline PA36 & Pseudomonas aeruginosa strain Y89 plasmid pY89 & 60.1 & 85842 & yes \\
\hline PA37 & Pseudomonas aeruginosa strain K34-7 plasmid pK34-7-1 & 30.1 & 4440 & yes \\
\hline PA38 & Pseudomonas aeruginosa strain AR441 plasmid unnamed3 & 57.1 & 438529 & yes \\
\hline PA39 & $\begin{array}{l}\text { Pseudomonas aeruginosa strain AR_0353 plasmid } \\
\text { unnamed1 }\end{array}$ & 60.8 & 41559 & yes \\
\hline PA40 & $\begin{array}{l}\text { Pseudomonas aeruginosa strain AR_0356 plasmid } \\
\text { unnamed2 }\end{array}$ & 57.1 & 438531 & yes \\
\hline PA41 & $\begin{array}{l}\text { Pseudomonas aeruginosa strain D } 5170990 \text { plasmid } \\
\text { pD } 5170990\end{array}$ & 60.3 & 32424 & yes \\
\hline PA42 & Pseudomonas aeruginosa strain 163940 plasmid pTROUS1 & 56.4 & 42035 & yes \\
\hline PA43 & Pseudomonas aeruginosa strain 121156 plasmid pNECK1 & 63.2 & 28859 & yes \\
\hline PA44 & Pseudomonas aeruginosa plasmid pCB58 & 58.6 & 32207 & yes \\
\hline PA45 & Pseudomonas aeruginosa strain PAG5 plasmid pPAG5 & 56.3 & 513322 & yes \\
\hline PA46 & Pseudomonas aeruginosa strain PA121617 plasmid pBM413 & 56.4 & 423017 & yes \\
\hline PA47 & Pseudomonas aeruginosa strain 1160 plasmid p1160-VIM & 56.2 & 205426 & yes \\
\hline PA48 & $\begin{array}{l}\text { Pseudomonas aeruginosa strain ST463 plasmid p1011- } \\
\text { KPC2 }\end{array}$ & 58.8 & 62793 & yes \\
\hline PA49 & Pseudomonas aeruginosa strain PA-IMP-1 plasmid pYUI-1 & 58.2 & 21079 & yes \\
\hline PA50 & Pseudomonas aeruginosa strain ST1006 plasmid pPA-2 & 55.5 & 7995 & yes \\
\hline PA51 & Pseudomonas aeruginosa plasmid YLH6_p3 & 57.8 & 49162 & yes \\
\hline
\end{tabular}




\begin{tabular}{|lllll|}
\hline $\begin{array}{l}\text { strain } \\
\text { number }\end{array}$ & Organism- plasmid & $\begin{array}{l}\text { G+C } \\
\%\end{array}$ & size bp & AMR \\
\hline PA52 & $\begin{array}{l}\text { Pseudomonas aeruginosa strain FDAARG0S_570 plasmid } \\
\text { unnamed }\end{array}$ & 61.3 & 36032 & yes \\
\hline PA53 & Pseudomonas aeruginosa strain TC4411 plasmid pPWIS1 & 57 & 419683 & yes \\
\hline PA54 & Pseudomonas aeruginosa strain PA298 plasmid pBM908 & 56.9 & 395774 & yes \\
\hline PA55 & Pseudomonas aeruginosa strain BH6 plasmid pBH6 & 55.9 & 3652 & yes \\
\hline PA56 & Pseudomonas aeruginosa plasmid pMATVIM-7 & 65.8 & 24179 & yes \\
\hline PA57 & Pseudomonas aeruginosa strain 2047 plasmid pPA2047 & 60.6 & 43660 & yes \\
\hline PA58 & Pseudomonas aeruginosa strain C79 plasmid p1 & 58.1 & 40180 & yes \\
\hline PA59 & Pseudomonas aeruginosa strain AR439 plasmid unnamed2 & 56.9 & 437392 & yes \\
\hline PA60 & Pseudomonas aeruginosa strain CF39S plasmid pCF39S & 56.6 & 468631 & yes \\
\hline PA61 & Pseudomonas aeruginosa strain T2436 plasmid pBT2436 & 56.9 & 422811 & yes \\
\hline PA62 & Pseudomonas aeruginosa strain T2101 plasmid pBT2101 & 57 & 439744 & yes \\
\hline PA63 & Pseudomonas aeruginosa plasmid Birmingham IncP-alpha & 61.8 & 60099 & yes \\
\hline PA64 & Pseudomonas aeruginosa IncP-1alpha plasmid pBS228 & 59 & 89147 & yes \\
\hline PA65 & Pseudomonas aeruginosa PA96 plasmid p0Z176 & 57.6 & 500839 & yes \\
\hline PA66 & Pseudomonas aeruginosa PA01 plasmid pAMBL2 & 60.4 & 24133 & yes \\
\hline PA67 & Pseudomonas aeruginosa PA01 plasmid pAMBL1 & 63.5 & 26440 & yes \\
\hline
\end{tabular}

\section{AMR genes for different drug classes}

Detection of AMR gene has been done by screening the plasmid sequences in ResFinder database. This database provides testing the existing of the resistance gene by complete sequences that acquired horizontally. This database highly associated with phenotypic resistance detection[4, 5, 13]. For high occurrence of resistance gene identification, it was adjusted to default setting. 67 out of 94 plasmids sequences were qualified as AMR containing plasmids. 
Table 2

frequency of AMR gene in

\begin{tabular}{|lll|}
\hline Pseudomonas aeruginosa plasmids \\
\hline AMR types & & number \\
\hline MDR & negative & 29 \\
& positive & 38 \\
& Total & 67 \\
XDR & negative & 62 \\
\cline { 2 - 3 } & positive & 5 \\
\hline \multirow{2}{*}{ PDR } & Total & 67 \\
& negative & 65 \\
& positive & 2 \\
\hline & Total & 67 \\
\hline
\end{tabular}

For detected 9 classes of AMR genes as shown in Table 3, aminoglycosides $39.6 \%$ were highest rate. The next frequencies were beta-lactams (19\%), sulphonamides (14.5\%), and fluoroquinolones (10.1\%). For nine classes of antibiotics, 74 AMR gene were identified. Prevalent of sulphonamide resistance gene Sul 1 was 32 out of 277 gene as shown in Fig. 1. 
Table 3

Resistance to different classes of antimicrobial drugs and p-MAR index found in a set of plasmids, the 40 plasmids listed showed the presence of different number of AMR genes to 9 drug classes. (Aminoglycoside $=$

AG; Beta-lactam = BL; macrolide-lincosamide-streptogramin B = MLS; Phenicol = PH; Rifampicin = RP; Sulphonamide = SM; Tetracycline $=$ TC; Trimethoprim $=$ TP)

\begin{tabular}{|c|c|c|c|c|c|c|c|c|c|c|c|c|}
\hline$s / n$ & Organism/Plasmid & AG & BL & MLS & $\mathrm{PH}$ & FQ & RP & SM & $\mathrm{TC}$ & TP & Total & $\begin{array}{l}\text { (p-MAR) } \\
\text { index }\end{array}$ \\
\hline 1 & $\begin{array}{l}\text { Pseudomonas } \\
\text { aeruginosa IncP- } \\
\text { 1alpha plasmid } \\
\text { pBS228 }\end{array}$ & 1 & 1 & & & & & & 1 & 1 & 4 & 0.266667 \\
\hline 2 & $\begin{array}{l}\text { Pseudomonas } \\
\text { aeruginosa PA96 } \\
\text { plasmid p0Z176 }\end{array}$ & 8 & 4 & & 2 & 6 & & 2 & & & 22 & 0.333333 \\
\hline 3 & $\begin{array}{l}\text { Pseudomonas } \\
\text { aeruginosa PAO1 } \\
\text { plasmid pAMBL2 }\end{array}$ & 2 & 3 & & & 1 & & 1 & & & 7 & 0.266667 \\
\hline 4 & $\begin{array}{l}\text { Pseudomonas } \\
\text { aeruginosa } \\
\text { plasmid } \\
\text { Birmingham IncP- } \\
\text { alpha }\end{array}$ & 1 & 1 & & & & & & 1 & & 3 & 0.2 \\
\hline 5 & $\begin{array}{l}\text { Pseudomonas } \\
\text { aeruginosa } \\
\text { plasmid p12939- } \\
\text { PER }\end{array}$ & 6 & 2 & & & & & 2 & & & 10 & 0.2 \\
\hline 6 & $\begin{array}{l}\text { Pseudomonas } \\
\text { aeruginosa } \\
\text { plasmid p727-IMP }\end{array}$ & 2 & 2 & 2 & 1 & 2 & 1 & 1 & 1 & 1 & 13 & 0.6 \\
\hline 7 & $\begin{array}{l}\text { Pseudomonas } \\
\text { aeruginosa } \\
\text { plasmid pA681- } \\
\text { IMP }\end{array}$ & 3 & 3 & 2 & 1 & 2 & & 4 & & & 15 & 0.4 \\
\hline 8 & $\begin{array}{l}\text { Pseudomonas } \\
\text { aeruginosa } \\
\text { plasmid pBS228 }\end{array}$ & 1 & 1 & & & & & & 1 & 1 & 4 & 0.266667 \\
\hline 9 & $\begin{array}{l}\text { Pseudomonas } \\
\text { aeruginosa } \\
\text { plasmid pCB58 }\end{array}$ & 10 & 1 & & & 1 & & 1 & & & 13 & 0.266667 \\
\hline 10 & $\begin{array}{l}\text { Pseudomonas } \\
\text { aeruginosa } \\
\text { plasmid pJB12 }\end{array}$ & 5 & 1 & & & 1 & & 1 & & & 8 & 0.266667 \\
\hline 11 & $\begin{array}{l}\text { Pseudomonas } \\
\text { aeruginosa } \\
\text { plasmid pJB35 }\end{array}$ & 5 & 1 & & & 1 & & 1 & & & 8 & 0.266667 \\
\hline
\end{tabular}




\begin{tabular}{|c|c|c|c|c|c|c|c|c|c|c|c|c|}
\hline$s / n$ & Organism/Plasmid & AG & BL & MLS & $\mathrm{PH}$ & FQ & RP & SM & TC & TP & Total & $\begin{array}{l}\text { (p-MAR) } \\
\text { index }\end{array}$ \\
\hline 12 & $\begin{array}{l}\text { Pseudomonas } \\
\text { aeruginosa } \\
\text { plasmid pR31014- } \\
\text { IMP }\end{array}$ & 3 & 2 & 2 & 1 & 2 & 1 & 2 & 1 & 1 & 15 & 0.6 \\
\hline 13 & $\begin{array}{l}\text { Pseudomonas } \\
\text { aeruginosa } \\
\text { plasmid R1033 } \\
\text { transposon } \\
\text { Tn1696 }\end{array}$ & 2 & & & 1 & & & 1 & & & 4 & 0.2 \\
\hline 14 & $\begin{array}{l}\text { Pseudomonas } \\
\text { aeruginosa strain } \\
1160 \text { plasmid } \\
\text { p1160-VIM }\end{array}$ & 5 & 1 & 3 & 1 & 3 & & 2 & & & 15 & 0.4 \\
\hline 15 & $\begin{array}{l}\text { Pseudomonas } \\
\text { aeruginosa strain } \\
121156 \text { plasmid } \\
\text { pNECK1 }\end{array}$ & 1 & 2 & & & 1 & & 1 & & & 5 & 0.266667 \\
\hline 16 & $\begin{array}{l}\text { Pseudomonas } \\
\text { aeruginosa strain } \\
163940 \text { plasmid } \\
\text { pTROUS1 }\end{array}$ & 1 & 2 & & & 1 & & 1 & & & 5 & 0.266667 \\
\hline 17 & $\begin{array}{l}\text { Pseudomonas } \\
\text { aeruginosa strain } \\
\text { AR_0356 plasmid } \\
\text { unnamed2 }\end{array}$ & 4 & & & & 2 & & 2 & 2 & & 10 & 0.266667 \\
\hline 18 & $\begin{array}{l}\text { Pseudomonas } \\
\text { aeruginosa strain } \\
\text { AR439 plasmid } \\
\text { unnamed2 }\end{array}$ & 12 & 2 & & & & & 2 & & & 16 & 0.2 \\
\hline 19 & $\begin{array}{l}\text { Pseudomonas } \\
\text { aeruginosa strain } \\
\text { AR441 plasmid } \\
\text { unnamed3 }\end{array}$ & 4 & & & & 2 & & 2 & 2 & & 10 & 0.266667 \\
\hline 20 & $\begin{array}{l}\text { Pseudomonas } \\
\text { aeruginosa strain } \\
\text { CF39S plasmid } \\
\text { pCF39S }\end{array}$ & 8 & & & & 2 & & 2 & 2 & & 14 & 0.266667 \\
\hline 21 & $\begin{array}{l}\text { Pseudomonas } \\
\text { aeruginosa strain } \\
\text { D5170990 } \\
\text { plasmid } \\
\text { pD5170990 }\end{array}$ & 9 & 1 & & 1 & & & 1 & & & 12 & 0.266667 \\
\hline 22 & $\begin{array}{l}\text { Pseudomonas } \\
\text { aeruginosa strain } \\
\text { FDAARGOS_570 } \\
\text { plasmid unnamed }\end{array}$ & 14 & 6 & & 2 & 4 & & 2 & & & 28 & 0.333333 \\
\hline
\end{tabular}




\begin{tabular}{|c|c|c|c|c|c|c|c|c|c|c|c|c|}
\hline$s / n$ & Organism/Plasmid & AG & BL & MLS & $\mathrm{PH}$ & FQ & RP & SM & TC & TP & Total & $\begin{array}{l}\text { (p-MAR) } \\
\text { index }\end{array}$ \\
\hline 23 & $\begin{array}{l}\text { Pseudomonas } \\
\text { aeruginosa strain } \\
\text { FFUP_PS_37 } \\
\text { plasmid pJB37 }\end{array}$ & 5 & 1 & & & 1 & & 1 & & & 8 & 0.266667 \\
\hline 24 & $\begin{array}{l}\text { Pseudomonas } \\
\text { aeruginosa strain } \\
\text { HN39 plasmid } \\
\text { pHN39-SIM }\end{array}$ & 1 & 2 & 1 & 1 & & 1 & 1 & & & 7 & 0.4 \\
\hline 25 & $\begin{array}{l}\text { Pseudomonas } \\
\text { aeruginosa strain } \\
\text { IP40a plasmid } \\
\text { pIP40 }\end{array}$ & 1 & 1 & & & & & 1 & & & 3 & 0.2 \\
\hline 26 & $\begin{array}{l}\text { Pseudomonas } \\
\text { aeruginosa strain } \\
\text { MRSN17623 } \\
\text { plasmid } \\
\text { pMRVIM0713 }\end{array}$ & 7 & 3 & & 1 & 2 & & 1 & & & 14 & 0.333333 \\
\hline 27 & $\begin{array}{l}\text { Pseudomonas } \\
\text { aeruginosa strain } \\
\text { PA121617 plasmid } \\
\text { pBM413 }\end{array}$ & 6 & 4 & 4 & 2 & 4 & & 4 & & & 24 & 0.333333 \\
\hline 28 & $\begin{array}{l}\text { Pseudomonas } \\
\text { aeruginosa strain } \\
\text { PA298 plasmid } \\
\text { pBM908 }\end{array}$ & 4 & 6 & & 2 & 4 & & 2 & & & 18 & 0.333333 \\
\hline 29 & $\begin{array}{l}\text { Pseudomonas } \\
\text { aeruginosa strain } \\
\text { PA34 plasmid } \\
\text { pMKPA34-1 }\end{array}$ & 5 & 1 & & 1 & & & 1 & 1 & 1 & 10 & 0.4 \\
\hline 30 & $\begin{array}{l}\text { Pseudomonas } \\
\text { aeruginosa strain } \\
\text { PA41437 plasmid } \\
\text { pOXA-198 }\end{array}$ & 4 & 1 & & 1 & & & 1 & & & 7 & 0.266667 \\
\hline 31 & $\begin{array}{l}\text { Pseudomonas } \\
\text { aeruginosa strain } \\
\text { PAB546 plasmid } \\
\text { pNK546-KPC }\end{array}$ & 1 & 1 & & 1 & & & 1 & & & 4 & 0.266667 \\
\hline 32 & $\begin{array}{l}\text { Pseudomonas } \\
\text { aeruginosa strain } \\
\text { PABL048 plasmid } \\
\text { pPABL048 }\end{array}$ & 4 & 2 & & & & & 2 & & & 8 & 0.2 \\
\hline 33 & $\begin{array}{l}\text { Pseudomonas } \\
\text { aeruginosa strain } \\
\text { PAcoop101 } \\
\text { plasmid pCOOP- } \\
101\end{array}$ & 5 & 1 & & & & & 1 & & 3 & 10 & 0.266667 \\
\hline
\end{tabular}




\begin{tabular}{|c|c|c|c|c|c|c|c|c|c|c|c|c|}
\hline $\mathbf{s} / \mathbf{n}$ & Organism/Plasmid & AG & $B L$ & MLS & $\mathrm{PH}$ & $\mathrm{FQ}$ & $\mathrm{RP}$ & SM & $\mathrm{TC}$ & TP & Total & $\begin{array}{l}\text { (p-MAR) } \\
\text { index }\end{array}$ \\
\hline 34 & $\begin{array}{l}\text { Pseudomonas } \\
\text { aeruginosa strain } \\
\text { PAG5 plasmid } \\
\text { pPAG5 }\end{array}$ & 6 & 4 & 4 & 2 & 4 & 2 & 4 & & 2 & 28 & 0.533333 \\
\hline 35 & $\begin{array}{l}\text { Pseudomonas } \\
\text { aeruginosa strain } \\
\text { PB353 plasmid } \\
\text { pPB353_1 }\end{array}$ & 4 & 6 & & & 4 & & 2 & & & 16 & 0.266667 \\
\hline 36 & $\begin{array}{l}\text { Pseudomonas } \\
\text { aeruginosa strain } \\
\text { PB354 plasmid } \\
\text { pPB354_1 }\end{array}$ & 2 & 3 & & & 2 & & 1 & & & 8 & 0.266667 \\
\hline 37 & $\begin{array}{l}\text { Pseudomonas } \\
\text { aeruginosa strain } \\
\text { S04 } 90 \text { plasmid }\end{array}$ & 4 & 2 & & & & & 2 & & & 8 & 0.2 \\
\hline 38 & $\begin{array}{l}\text { Pseudomonas } \\
\text { aeruginosa strain } \\
\text { T2101 plasmid } \\
\text { pBT2101 }\end{array}$ & 8 & 12 & & & & & 8 & 4 & & 32 & 0.266667 \\
\hline 39 & $\begin{array}{l}\text { Pseudomonas } \\
\text { aeruginosa strain } \\
\text { T2436 plasmid } \\
\text { pBT2436 }\end{array}$ & 10 & 4 & & 2 & & 2 & 4 & 2 & & 24 & 0.4 \\
\hline 40 & $\begin{array}{l}\text { Pseudomonas } \\
\text { aeruginosa strain } \\
\text { TC4411 plasmid } \\
\text { pPWIS1 }\end{array}$ & 5 & 1 & & & & & 1 & & & 7 & 0.2 \\
\hline
\end{tabular}

\section{Antibiotic resistance determinants in Aeromonas plasmids}

In AMR plasmids, 6 ARD family were detected. High frequency families were aminoglycosides acetyltransferases and beta-lactamase with $38.30 \%$ and $31.91 \%$, respectively as shown in Fig. 2 .

\section{phylogenetic analysis of plasmids}

Complete genome sequences of 67 plasmid containing AMR genes were analyzed using software with consideration exclusion and inclusion criteria. Representative sequence from each group were selected with maximum parsimony tree approach. Finally, 15 representative genomic sequences selected from each clade were more analyzed (Fig. 3). As shown in Fig. 3 the evolutionary lineages and common ancestry of all plasmid sequences were revealed by the phylogenetic tree. Three clades were revealed from them. The relationship of clades with drug resistance was not significant $(p-v a l u e=0.682)$ as shown in Table 4. 
Table 4

Distribution of MDR based on clades ( $p$ -

value $=0.682$ )

\begin{tabular}{|c|c|c|c|}
\hline & MDR & & \\
\hline Clade & no & yes & Total \\
\hline 1 & $13.33 \%$ & $20.00 \%$ & $33.33 \%$ \\
\hline 2 & $20.00 \%$ & $6.67 \%$ & $26.67 \%$ \\
\hline 3 & $20.00 \%$ & $20.00 \%$ & $40.00 \%$ \\
\hline Total & $53.33 \%$ & $46.67 \%$ & $100.00 \%$ \\
\hline
\end{tabular}

\section{Statistical analysis of sequences}

Online tools like as NCBI database, ResFinder 2.1 database/webserver, KmerResistance 2.2 database, ResFinderFG 1.0. and MEGA X Molecular Evolutionary Genetics Analysis were used for analyzing sequences and resistance gene. XLSTAT were used for PCA and statistical analysis.

\section{Discussion}

Antibiotic resistance is a result of selection pressure due to the overuse and frequent misuse of antibiotics in health systems. The profile of AMR gene in bacterial population is depend on horizontal transfer of mobile genetic elements. Plasmids are one of the major roles as mobile genetic elements. Specification of plasmid in bacterial give more insight in determination of ability of plasmid to transfer resistance gene and evaluation of genome. Pseudomonas aeruginosa plasmids has been sequenced and evaluated statistically, based on PCA analysis there was the negative Pearson's correlation $(r=-0.191, a=0.95)$ between GC content and plasmid size. The negative correlation between plasmid specification may be due random or naturally transfer of genes among plasmids. Although there is correlation between genome size and guanine-cytosine (GC) content in bacteria, however, the this correlation not well explained [14].

The prevalence of AMR genes was screened in ResFinder database. Plasmids sequences were analyzed to detect acquired antimicrobial resistance genes. Disadvantage of this way is that the ResFinder database cannot find mutation, therefore it only detects acquired resistance genes. There is need to qualify resistance genotype with phenotypic identification. Investigation showed that there is very high correlation between phenotypic detection and AMR gene detection in ResFinder database, therefore, whole-genome sequences alignment is an alternative way to find drug resistance patterns [15]. In overall $71.28 \%$ of plasmid were containing AMR genes, of which, 38, 5, 2 isolates were MDR, XDR and PDR respectively.

The gene responsible for this resistance phenotype were aminoglycosides $39.6 \%$, beta-lactams (19\%), sulphonamides (14.5\%), and fluoroquinolones (10.1\%). For nine classes of antibiotics, 74 AMR gene were identified. Prevalent of sulphonamide resistance gene Sul1 was 32 out of 277 genes. Aminoglycosides are used for the treatment of Pseudomonas aeruginosa infections such as pulmonary infections in cystic fibrosis (CF) patients. This antibiotic is inactivated by enzymes that phosphorylate (aminoglycoside 
phosphoryltransferase [APH]), acetylate (aminoglycoside acetyltransferase [AAC]), or adenylate (aminoglycoside nucleotidyltransferase [ANT] of which acetylation of aminoglycosides mainly occur at the 1 , $3,6^{\prime}$, and $2^{\prime}$ amino groups. The aminoglycosides resistance genes also transferred by means of plasmids[16]. The Sul1 gene located in plasmids and products of this gene can degrade sulfonamides and trimethoprim antibiotics. Those are cheap and efficient antibiotics that have been used for a long time to treatment of human and animals' gram-negative infections [17]. Three genes sul1, sul 2 and sul 3 encode dihydropteroate synthase enzyme that inactivate sulfonamides and sul1 gene is mostly transferred in associated with class 1 integrons and others by plasmids [18]. Quinolone resistance has been occur mostly by plasmids by tree mechanisms including (i) qnrgenes that produce a quinolone-protective proteins, (ii) aac(6)-lb gene that produce a double class antibiotic-modifying enzyme which acetylates ciprofloxacin and norfloxacin and (iii) qepA gene that produce an efflux pump proteins [19].

Analyzing the Information in annotated sequence is reveal the mechanism of spreading of resistance gene in plasmids. Detection the trace of AMR genes in world papulation can help to find more response to question in regards to spread of infection and analysis of AMR gene sequences give more insight to scientist to control of Pseudomonas aeruginosa infections. MEGA X software were used for analyzing phylogenetic relationship between 67 plasmid containing AMR genes. Due to some restriction test were accomplished in three group and representative genomic sequences from each clade were more analyzed. Finally, three clades were revealed from analyzing representative sequences. There was no correlation of MDR with clades ( $p$-value = 0.682)

\section{Conclusion}

This study results showed that there is no correlation between size and GC content of Pseudomonas aeruginosa plasmids. Most of plasmid carrying AMR genes that acquired horizontally. Three phylogenetically clade was revealed by molecular epidemiology software but there were not associated with drug resistance.

\section{Declarations}

\section{Ethical Approval and Consent to participate}

Not applicable

\section{Consent for publication}

Not applicable

\section{Availability of data and materials}

Not applicable

\section{Competing interests}

The authors have no conflict of interest. 


\section{Funding}

Not applicable

\section{Authors' contributions}

RR did all process including doing project and manuscript writing, editing and submitting.

\section{Acknowledgements}

The authors thank research deputy of Ardabil university for supporting this project.

\section{Authors' information}

Rashid Ramazanzadeh professor of medical bacteriology in Ardabil university of medical sciences.

\section{Conflict of interest}

The authors have no conflict of interest.

\section{References}

1. Sevastsyanovich, Y.R., et al., Diversity of IncP-9 plasmids of Pseudomonas. Microbiology (Reading, England), 2008. 154(Pt 10): p. 2929-2941.

2. Bardaji, L., et al., Plasmid Replicons from Pseudomonas Are Natural Chimeras of Functional, Exchangeable Modules. Front Microbiol, 2017. 8: p. 190.

3. Thomas, C.M. and C.A. Smith, Incompatibility Group P Plasmids: Genetics, Evolution, and Use in Genetic Manipulation. Annual Review of Microbiology, 1987. 41(1): p. 77-101.

4. Nwaiwu, O. and C.C. Aduba, An in silico analysis of acquired antimicrobial resistance genes in Aeromonas plasmids. AIMS Microbiol, 2020. 6(1): p. 75-91.

5. Zankari, E., et al., Identification of acquired antimicrobial resistance genes. Journal of antimicrobial chemotherapy, 2012. 67(11): p. 2640-2644.

6. Davis, R. and P.D. Brown, Multiple antibiotic resistance index, fitness and virulence potential in respiratory Pseudomonas aeruginosa from Jamaica. J Med Microbiol, 2016. 65(4): p. 261-271.

7. Krumperman, P.H., Multiple antibiotic resistance indexing of Escherichia coli to identify high-risk sources of fecal contamination of foods. Appl Environ Microbiol, 1983. 46(1): p. 165-70.

8. Magiorakos, A.P., et al., Multidrug-resistant, extensively drug-resistant and pandrug-resistant bacteria: an international expert proposal for interim standard definitions for acquired resistance. Clin Microbiol Infect, 2012. 18(3): p. 268-81.

9. Sandhu, R., S. Dahiya, and P. Sayal, Evaluation of multiple antibiotic resistance (MAR) index and Doxycycline susceptibility of Acinetobacter species among inpatients. Indian Journal of Microbial Research, 2016. 3: p. 299-304. 
10. Clausen, P.T., et al., Benchmarking of methods for identification of antimicrobial resistance genes in bacterial whole genome data. J Antimicrob Chemother, 2016. 71(9): p. 2484-8.

11. Clausen, P., F.M. Aarestrup, and O. Lund, Rapid and precise alignment of raw reads against redundant databases with KMA. BMC Bioinformatics, 2018. 19(1): p. 307.

12. Center for Genomic Epidemiology ResFinderFG 1.0 database. Available from: https://cge.cbs.dtu.dk/services/ResFinderFG.

13. Zhang, Z., et al., A greedy algorithm for aligning DNA sequences. Journal of Computational biology, 2000. 7(1-2): p. 203-214.

14. Nishida, H., Evolution of genome base composition and genome size in bacteria. Frontiers in microbiology, 2012. 3: p. 420.

15. Zankari, E., et al., Genotyping using whole-genome sequencing is a realistic alternative to surveillance based on phenotypic antimicrobial susceptibility testing. J Antimicrob Chemother, 2013. 68(4): p. 771-7.

16. Poole, K., Aminoglycoside Resistance in <i>Pseudomonas aeruginosa</i>. Antimicrobial Agents and Chemotherapy, 2005. 49(2): p. 479-487.

17. Sköld, O., Resistance to trimethoprim and sulfonamides. Vet Res, 2001. 32(3-4): p. 261-73.

18. Domínguez, M., et al., Occurrence of Transferable Integrons and sul and dfr Genes Among Sulfonamideand/or Trimethoprim-Resistant Bacteria Isolated From Chilean Salmonid Farms. Front Microbiol, 2019. 10: p. 748.

19. Quiroga, M.P., et al., Complex class 1 integrons with diverse variable regions, including aac(6')-Ib-cr, and a novel allele, qnrB10, associated with ISCR1 in clinical enterobacterial isolates from Argentina. Antimicrobial agents and chemotherapy, 2007. 51(12): p. 4466-4470.

\section{Figures}




\section{Total}

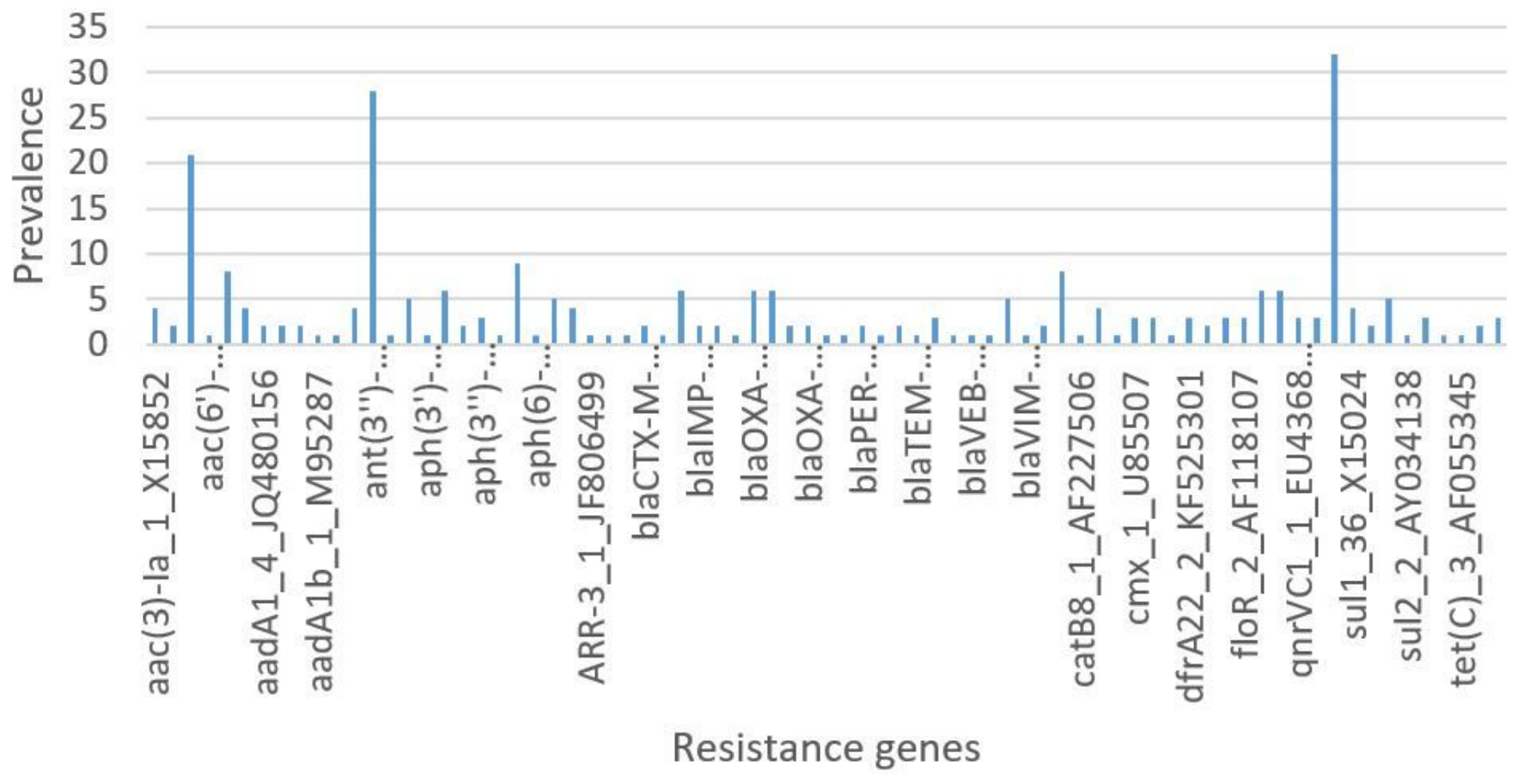

Figure 1

Prevalence of 74 AMR genes found in 40 Aeromonas plasmids after in silico analysis 


\section{Total}

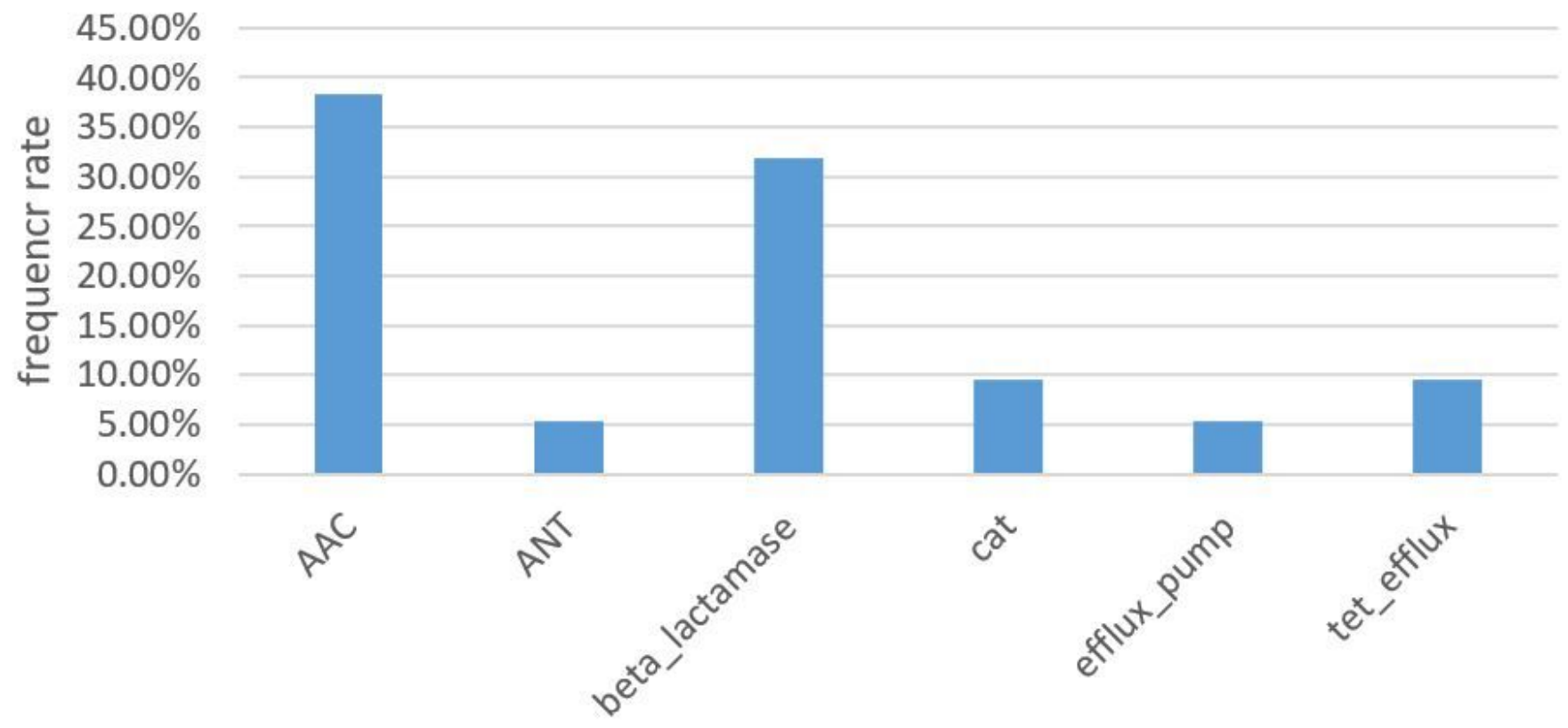

Antibiotics Family

\section{Figure 2}

Frequency of Antibiotics family in AMS containing plasmids 


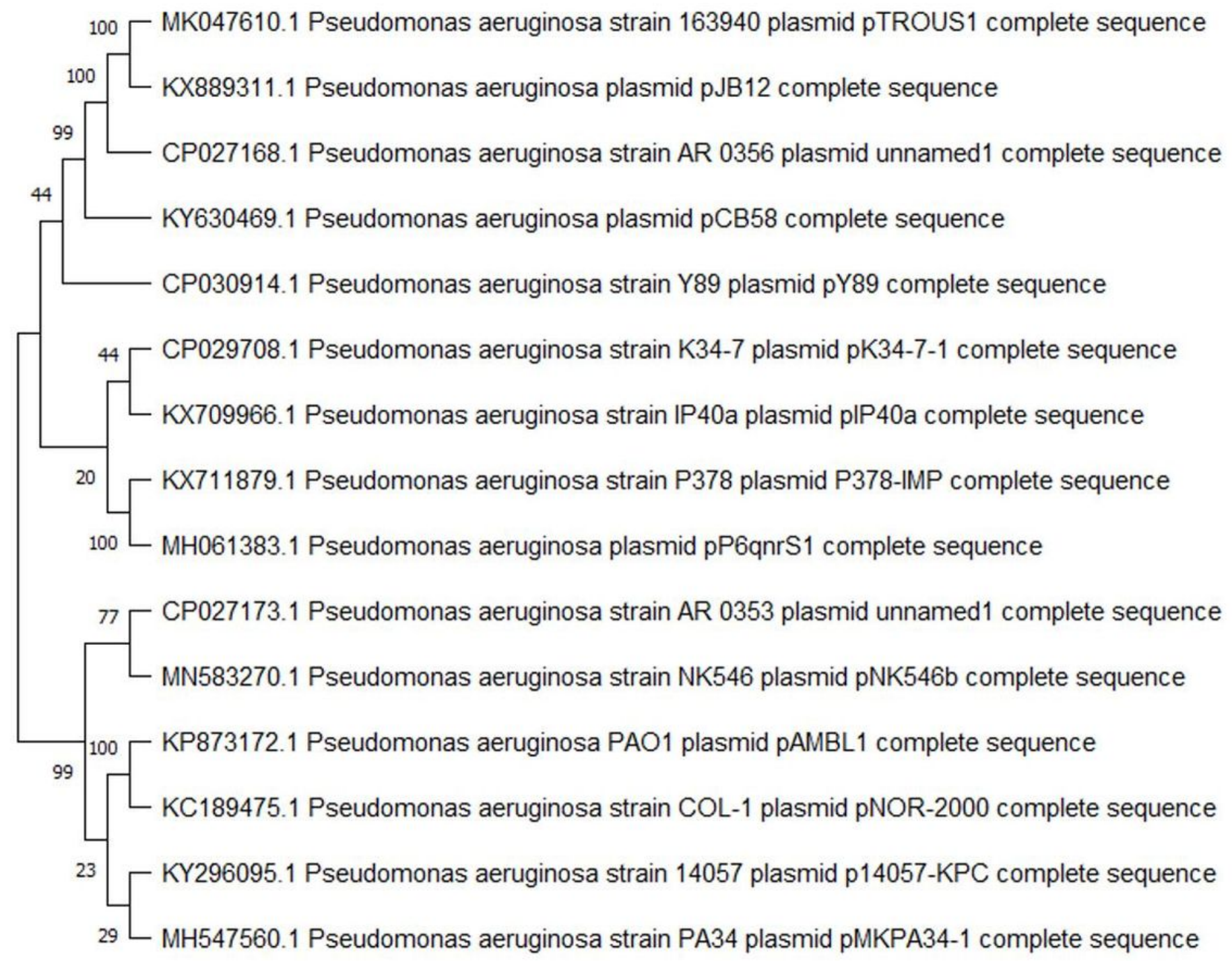

\section{Figure 3}

The phylogenetic analysis of 15 complete genome sequence of representative Pseudomonas aeruginosa plasmids by Maximum parsimony method using 1,000 bootstraps. The scale represents 0.1 substitutions per nucleotide position all of the accession numbers and full name of the strains were listed. 